\title{
A Report of Two Cases of Cerebral Toxoplasmosis in Leukemia Patients
}

\author{
Mohammad Pedram ${ }^{1}$, Sharif Maraghi ${ }^{2, *}$, Ahmad Soltani Shirazi ${ }^{3}$, Kaveh Jaseb ${ }^{1}$, Sabahat \\ Haghi $^{1}$ \\ ${ }^{1}$ Shafa Hospital and Research Center of Thalassemia and Haemoglobinopathy, Jundishpur University of Medical Sciences, Ahvaz, IR Iran \\ ${ }^{2}$ Department of Parasitology and Mycology, Abadan Arvand International Division, Infectious and Tropical Diseases, Thalassemia and Haemoglobinopathy Research Centers, \\ Jundishapur University of Medical Sciences, Ahvaz, IR Iran \\ ${ }^{3}$ Radiology Department, School of Medicine, Jundishapur University of Medical Sciences, Ahvaz, IR Iran \\ ${ }^{*}$ Corresponding author: Sharif Maraghi, Department of Parasitology and Mycology, Abadan Arvand International Division, Ahvaz Jundishapur University of Medical Sciences, Ahvaz, \\ IR Iran. Tel: +98-9161184914, Fax:+98-6112231325, E-mail: maraghis@gmail.com.
}

Received: November 11, 2012; Revised: December 1, 2012; Accepted: December 9, 2012

\begin{abstract}
Introduction:: Toxoplasmosis is a parasitic infection cause by Toxoplasma gondii. This parasite is associated with congenital infection and can cause abortion, encephalitis or systemic infection in immune- compromised patients.

Case Presentation: In this study, two cases of central nervous system toxoplasmosis in leukemia patients are reported. The first case was a 15-year-old girl with acute lymphoblastic leukemia (ALL) who was using maintenance treatment for one year. The patient referred in 2010 to the hospital with headache, nausea and vomiting since three weeks ago. Brain magnetic resonance imaging (MRI) indicated a hypointense lesion in the left temporal compatible with cerebral toxoplasmosis. Serum immunoglobulin M(Ig M) rate against Toxoplasma was 8 and Ig $\mathrm{G}$ was $5 \mathrm{IU} / \mathrm{mL}$. The patient was prescribed with pyrimethamin, sulfadiazine and lecoverin, but she was expired eight months after toxoplasmosis infection with bone marrow relapse in re- induction period of chemotherapy. The second case was a 6-year-old boy with acute myeloblastic leukemia (AML) diagnosed in 2010. The patient was on maintenance treatment. Two months ago, he was admitted to a hospital because of high temperature. In the brain MRI a $30 \mathrm{~mm}$ mass was revealed. Ig M antibody was more than 9 and Ig G was 6 IU/ $\mathrm{mL}$. The patient received pyrimethamin, sulfadiazine and leucoverin. He is alive and has the neurologic sequel.

Conclusions: Disseminated toxoplasmosis is a major health problem in immune- compromised patients, and it is necessary to examine the patients before, during and after chemotherapy for toxoplasmosis.
\end{abstract}

Keywords: Toxoplasmosis; Leukemia; Immune- Compromise; Brain; Iran

\section{Introduction}

Toxoplasma gondii is known as one of the most common infectious protozoan parasites that has a worldwide distribution (1-3). Cats are recognized as the only definitive host of $T$. gondii, but humans and other warmed-blooded animals and birds are as intermediate hosts. Humans can be infected by ingestion of raw or uncooked meat containing tissue cysts, unwashed vegetables, contaminated water or soil. Congenital toxoplasmosis occurs via the trans-placental route (4). Toxoplasma infection is largely asymptomatic, but in those individuals who are immune-compromised with AIDS, malignant patients under chemotherapy or organ transplant recipients can become disseminated and cause severe toxoplasmosis and/or encephalitis (5-7). In the present article two cases of cerebral toxoplasmosis in leukemia patients from Ahvaz, southwest Iran is reported.

\section{Case Report}

Case 1: A 15-year-old girl with acute lymphoblastic leukemia diagnosed in 2010 was receiving maintenance treatment. Three weeks ago, she referred to a hospital with headache, nausea and vomiting. CT scan of the brain demonstrated low density of mass like a lesion of the left parietal lobes cortico medullary junction accompanies with mild vasogenic edema and no enhancement after contrast media administration. Findings were non specific in CT scan. MRI was applied and on the obtained images there was left parietal corticomedullary junction intermediate signal intensity lesion with vasogenic edema on $\mathrm{T} 2$ which as a bright intermediated signal in $\mathrm{T} 1$ and low signal intensity peripheral edema (Figure 1A, 1B), follow in contrast media administration remarkable enhancements of lesions are seen (Figure 1C). These findings were compatible with toxoplasmosis and lymphoma. MRI spectroscopy (MRS) was done and increasing of lactate

Implication for health policy/practice/research/medical education:

Disseminated toxoplasmosis is a major health problem in immune- compromised patients, and it is necessary to examine the patients before, during and after chemotherapy for toxoplasmosis.

Copyright (C) 2013, Ahvaz Jundishapur University of Medical Sciences; Published by Kowsar Corp. This is an open-access article distributed under the terms of the Creative Commons Attribution License, which permits unrestricted use, distribution, and reproduction in any medium, provided the original work is properly cited. 

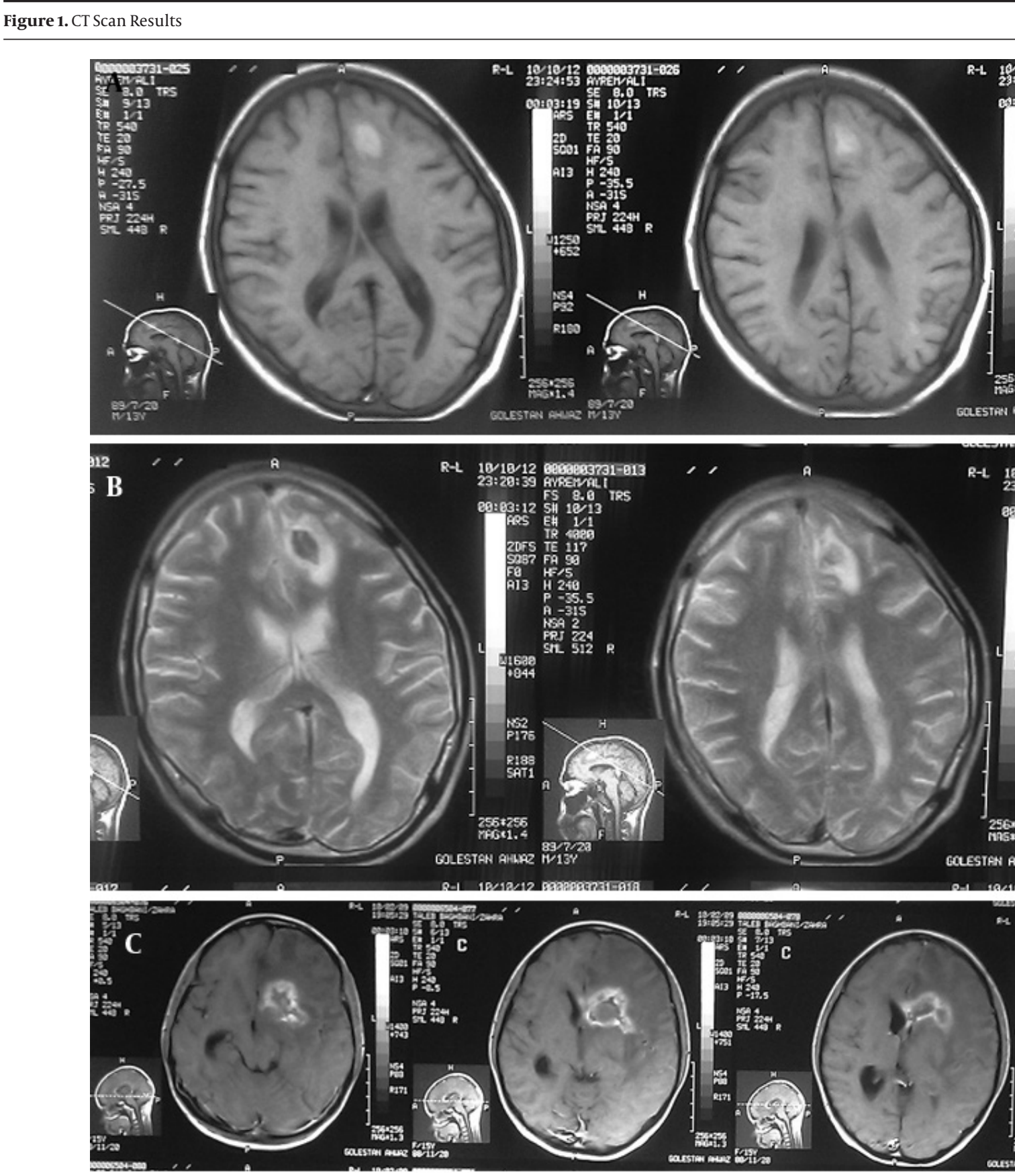

A:T1- Weighted axial MRI: Left parietal corticomedullary junction, bright intermediated signal and low signal intensity peripheral edema. B: T2-Weighted axial MRI: Left parietal corticomedullary junction, intermediate signal intensity lesion with vasogenic edema. C: MRI with contrast shows remarkable enhancement of lesions. 


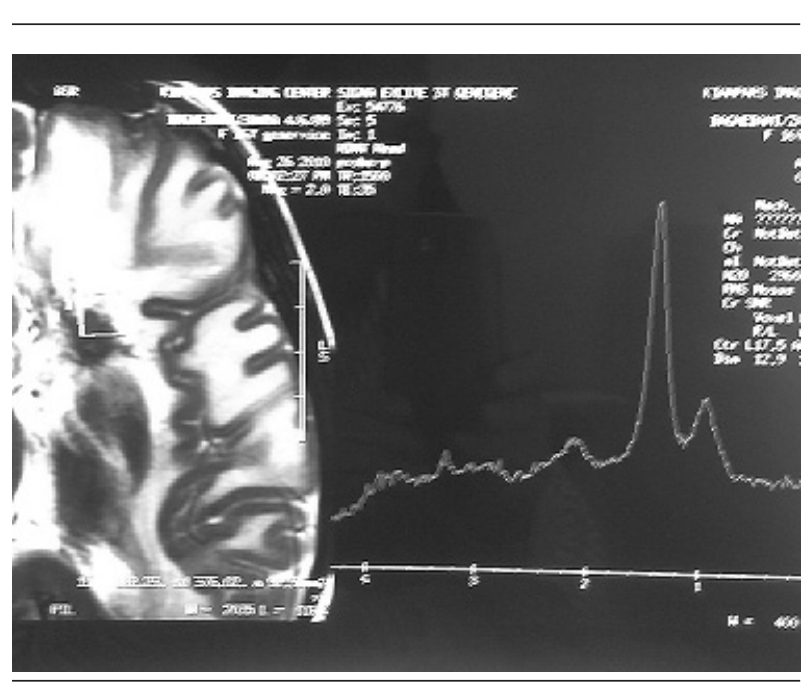

Figure 2. MR Spectroscopy: Increasing Lactate and Lipid and Low Choline Which Are Specific for Necrotizing Abscess.

Ig M antibody against Toxoplasma by Chemiluminesans (Roche kit) was $8 \mathrm{IU} / \mathrm{mL}$ (reference range: non reactive < 0.8 ; intermitant $0.8-1$; reactive $>1$ ) and $\mathrm{Ig} \mathrm{G}$ was $4 \mathrm{IU} / \mathrm{mL}$ (reference range: Non reactive $<1$; intermitant 1 - 3 ; reactive $>3$ ). The patient prescribed with pyrimethamin, sulfadiazine and leucoverin.

The mass was operated and sample sent to pathology department. Histopathological examination revealed necrotic inflammatory lesion, Ziehl- Nelson and PAS staining were negative for acid- fast bacilli and fungi, compatible with toxoplasmosis. The patient expired eight months after toxoplasmosis infection with bone marrow relapse in re- induction period of chemotherapy.

Case 2: A six-year-old boy with acute myeloblastic leukemia (AML) diagnosed in 2010 and was on maintenance treatment. Two months ago he was admitted to a hospital because of fever. Administration of antibacterial, antifungal and antiviral medicine did not control the temperature and due to generalized status tonic clonic convulsion, patient transferred into the intensive care unit (ICU).

Serological examination for anti- Toxoplasma antibody (Ig M) rate by Chemiluminesans (Roche kit) was more than 9 and Ig G was 6 IU/mL. Brain MRI revealed marked communicating hydrocephaly with corticomedullary junction of both temporal. Hyperdense lesions on T1 with surrounding vasogenic edema and intermediate signal on T2 with bright signal vasogenic edema and on post contrast images multiple enhancing lesions were seen (Figure 3). The patient received pyrimethamin, sul- fadiazine and leucovorin. The patient is alive and has neurogic sequel.

\section{Conclusions}

T. gondii infections in most of the immune- competent humans are asymptomatic, but can cause acute infection in immunosuppressed patients and congenital toxoplasmosis. It has been known that $15-58 \%$ of humans are infected with T. gondii, but the rate of infection varies due to many factors (8). Disease in immune- compromised individuals (i.e. persons with AIDS, transplant recipients, immunosuppressive drug users) usually due to reactivation of latent infection and can lead to lethal meningoencephalitis, focal lesion of CNS and less commonly, myocarditis or pneumonitis. The clinical pictures of cerebral toxoplasmosis may include headache, seizures, mental status changes, focal neurologic signs and aseptic meningitis (9).

In this presentation, two patients with ALL and AML under maintenance treatment were suffering from cerebral toxoplasmosis confirmed by determination of Ig M antibody against Toxoplasma and MRI. Both patients were using maintenance treatment for more than one year and chemotherapy cause the weakening of immune system and reactivation of latent infection. The first patient expired eight months after toxoplasmosis infection, and the second patient is still alive with the sequel. Ghasemian et al. in 2007 evaluated the anti Toxoplasma antibodies (Ig G and Ig M) in 252 cancer patients in Ahvaz and indicated that $15 \%$ of the cases were Ig G positive and $10.3 \%$ were positive for anti- Toxoplasma Ig $\mathrm{M}$ and $6.7 \%$ of cases revealed seropositivity for Ig $\mathrm{G}$ and Ig $M$ antibodies (10).

Toxoplasmosis has been reported in immune- compromised patients with lymphatic leukemia $(11,12)$, nonhodgkine lymphoma (13), bone marrow transplantation $(14)$, AIDS $(15,16)$, and means that if the immune system declines, the tissue cysts may reactivate and cause disseminated infection that manifests as encephalitis, myocarditis or chorioretinitis and can be fetal for immune-compromised patients $(17,18)$. Although imaging usually shows the hypointensity lesion on T2 and hyperintensive on T1, but Toxoplasma PCR is a useful technique for diagnosis of toxoplasmosis in blood and aqueous humor in patients with HIV, chorioretinitis, pulmonary, cerebral and congenital toxoplasmosis (19, 20). Disseminated toxoplasmosis is a major health problem in immune-compromised patients and it is necessary to examine the patients before, during and after chemotherapy for toxoplasmosis. 
Figure 3. Brain MRI: Marked Communicating Hydrocephaly With Corticomedullary Junction of Both Temporal
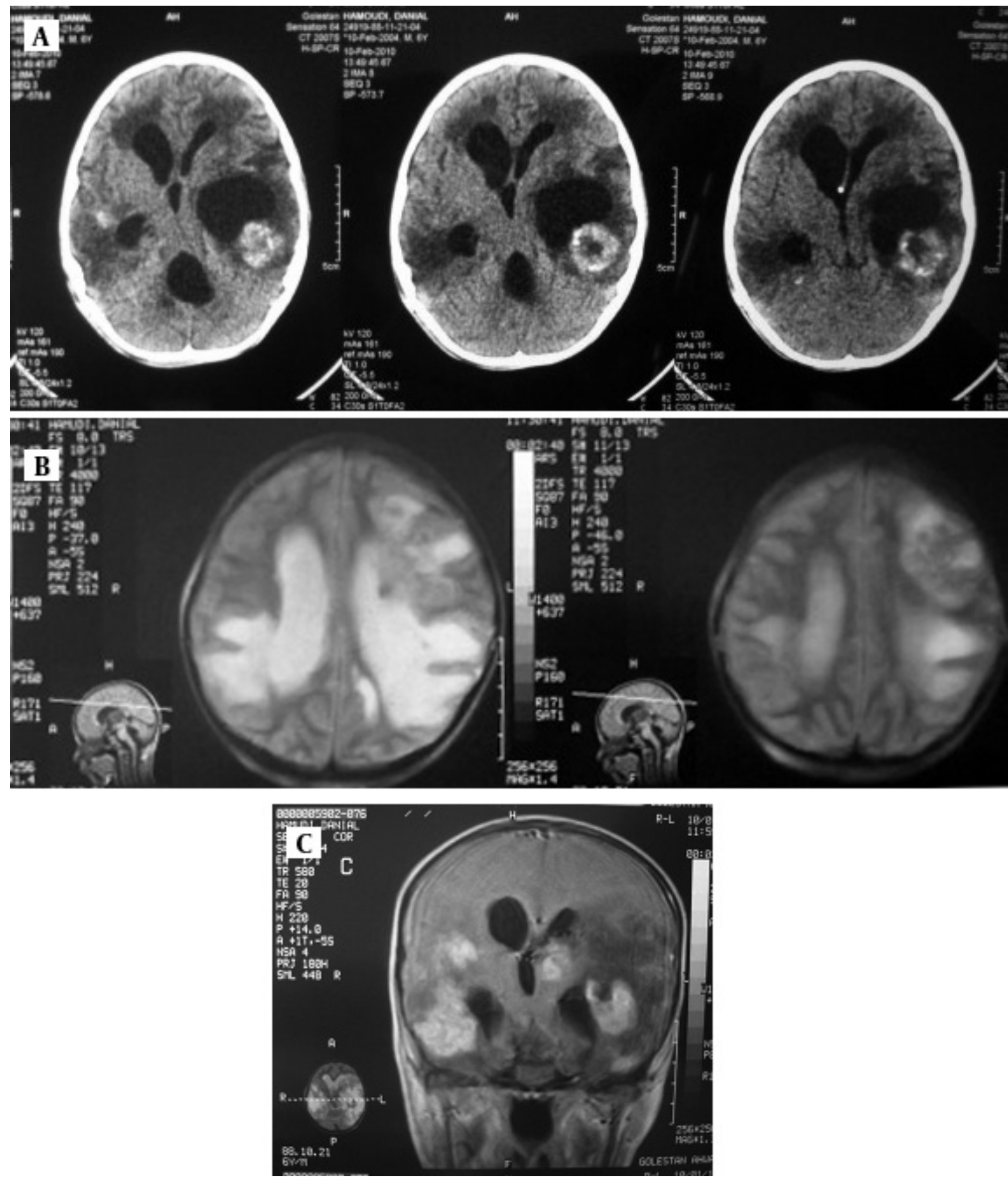

A:T1-Weighted axial MRI: Hyperdense lesions with surrounding vasogenic edema. B: T2-Weighted axial MRI: Intermediate signals with bright signal vasogenic edema. C: MRI with contrast: Multiple enhancing lesions.

\section{Acknowledgements}

We would like to thank Department of Radiology in
Golestan Hospital and Iran- Zamin Diagnostic Laboratory in Ahvaz and Thalassemia and Haemoglobinopathy 
Research Center of Jundishapur University of Medical Sciences in Iran for their critical helps.

\section{Authors' Contribution}

None declared.

\section{Financial disclosure}

The authors declare no financial disclosure.

\section{Funding/Support}

None declared.

\section{References}

1. Boothroyd John C, Grigg Michael E. Population biology of Toxoplasma gondii and its relevance to human infection: do different strains cause different disease? Curr Opin Microbiol. 2002;5(4):438-442.

2. Dubey JP. The history of Toxoplasma gondii-the first 100 years. $J$ Eukaryot Microbiol. 2008;55(6):467-75.

3. Petersen E. Toxoplasmosis. Semin Fetal Neonatal Med. 2007;12(3):214-23.

4. Kasper L, Courret N, Darche S, Luangsay S, Mennechet F, Minns L, et al. Toxoplasma gondii and mucosal immunity. Int J Parasitol. 2004;34(3):401-9.

5. Gallino A, Maggiorini M, Kiowski W, Martin X, Wunderli W, Schneider J, et al. Toxoplasmosis in heart transplant recipients. Eur J Clin Microbiol Infect Dis. 1996;15(5):389-93.

6. Velimirovic B. Toxoplasmosis in immunosuppression and AIDS. Infection. 1984;12(5):315-317.

7. Wanke C, Tuazon CU, Kovacs A, Dina T, Davis DO, Barton N, et al. Toxoplasma encephalitis in patients with acquired immune deficiency syndrome: diagnosis and response to therapy. Am J Trop Med Hyg. 1987;36(3):509-16.
8. Walzer P, Genta Robert M. Parasitic infections in the compromised host.: CRC Press; 1989.

9. Ho-Yen DO. Clinical features. Joss AWL editor. : Oxford: Oxford University Press; 1992.

10. Ghasemian M, Maraghi Sh, Saki J, Pedram M. Determination of Antibodies (IgG, IgM) against Toxoplasma gondii in Patients with Cancer. Iran J Parasitol. 2007;2(4):1-6.

11. Abedalthagafi M, Rushing EJ, Garvin D, Cheson B, Ozdemirli M. Asymptomatic diffuse "encephalitic" cerebral toxoplasmosis in a patient with chronic lymphocytic leukemia: case report and review of the literature. Int J Clin Exp Pathol. 2009;3(1):106-9.

12. Bacchu S, Fegan C, Neal J. Cerebral toxoplasmosis in a patient with chronic lymphocytic leukaemia treated with fludarabine. BrJ Haematol. 2007;139(3):349.

13. Herold MA, Kuhne R, Vosberg M, Ostheeren-Michaelis S, Vogt P, Karrer U. Disseminated toxoplasmosis in a patient with nonHodgkin lymphoma. Infection. 2009;37(6):551-4.

14. Mele A, Paterson PJ, Prentice HG, Leoni P, Kibbler CC. Toxoplasmosis in bone marrow transplantation: a report of two cases and systematic review of the literature. Bone Marrow Transplant. 2002;29(8):691-8.

15. Mariuz P, Steigbigel RT. Toxoplasma infection in HIV-infected patients. Toxoplasmosis A comprehensive clinical guide. 2001:147-177.

16. Sell M, Klingebiel R, Di Iorio G, Sampaolo S. Primary cerebral toxoplasmosis: a rare case of ventriculitis and hydrocephalus in AIDS. Clin Neuropathol. 2005;24(3):106-11.

17. Montoya JG, Liesenfeld O. Toxoplasmosis. Lancet. 2004;363(9425):1965-76.

18. Tenter AM, Heckeroth AR, Weiss LM. Toxoplasma gondii: from animals to humans. Int J Parasitol. 2000;30(12-13):1217-58.

19. Bou G, Figueroa MS, Marti-Belda P, Navas E, Guerrero A. Value of PCR for detection of Toxoplasma gondii in aqueous humor and blood samples from immunocompetent patients with ocular toxoplasmosis. J Clin Microbiol. 1999;37(11):3465-8.

20. Lamoril J, Molina JM, de Gouvello A, Garin YJ, Deybach JC, Modai J, et al. Detection by PCR of Toxoplasma gondii in blood in the diagnosis of cerebral toxoplasmosis in patients with AIDS.J Clin Pathol.1996;49(1):89-92. 